\title{
On Cardinality of the Group of Weak Fuzzy Automaton Isomorphisms
}

\author{
S. S. Dhure \\ Department of Mathematics \\ Shivaji University, Kolhapur(M.S.)-416 004
}

\author{
S. R. Chaudhari \\ Department of Mathematics \\ School of Mathematical Sciences \\ North Maharashtra University, Jalgaon (M.S.) - 425001
}

\begin{abstract}
Recent studies on fuzzy automata are influenced by algebraic techniques to tackle issues like reduction, minimization and their languages. Fuzzy automaton homomorphism is one such majorally discussed technique. This paper is concerned with the group of (weak) fuzzy automaton automorphisms and constructions of all (weak) fuzzy automaton automorphisms over arbitrary fuzzy automaton. It is shown that (1) every arbitrary fuzzy automaton is decomposed into distinct primaries, (2) primaries are maximal singly generated fuzzy automata and (3) every weak fuzzy automaton homomorphism on an arbitrary fuzzy automaton is uniquely determined into weak fuzzy automaton homomorphisms on all its primaries. Therefore, the discussion begun over strongly connected fuzzy automaton and continue constructions as well as characterizations of (weak) fuzzy automaton homomorphisms, isomorphisms, endomorphisms and automorphisms sequentially over perfect fuzzy automaton, singly generated fuzzy automaton and primaries of fuzzy automaton. Finally, it is obtained that the group of weak fuzzy automaton automorphisms and its cardinality over arbitrary fuzzy automaton.
\end{abstract}

\section{Keywords:}

Fuzzy function, fuzzy automaton (strongly connected, perfect and singly generated), (Weak) fuzzy automaton automorphism (homomorphism, isomorphism), primaries and basis of a fuzzy automaton.

\section{INTRODUCTION}

In many recent literature ([2],[4],[5],[8], [10],[13],[14],[15],,[16],[17]), researchers have discussed fuzzy automaton algebraically. It seems that the main motto behind them was to discuss algebraic properties of fuzzy automata in relation to their structure (i.e. fuzzy transition function) ([5],[11],[12], [16],[18], [20]). Structure preserving fuzzy transition functions isomorphisms in general and homomorphisms in particular - plays crucial role for equivalent and reduction of a fuzzy automaton([10], [11],[12], [14],[15],[16],[17]). The aim of this paper is to construct all elements of the group of (weak) fuzzy automaton automorphisms. We first discuss the nature of (weak) fuzzy automaton automorphisms through a string of inputs (called h-identifier) and used it to calculate the cardinality of the group.
It is pointed out, in remark 2.7, that the fuzzy automaton discussed in $([9],[11],[14],[16],[17])$ is unable to derive homomorphism between fuzzy automata corresponding to an identifier. Hence, throughout this paper we used fuzzy automaton, discussed in [10], based on the notion of fuzzy function. In section 2, it is shown that the set of all (weak) fuzzy automaton automorphisms forms a group. It is established that the cardinality of this group cannot exceed the number of states for a strongly connected fuzzy automaton. In section 3, we have discussed abelian and weakly abelian fuzzy automaton. It is proved that a fuzzy automaton is weakly abelian if and only if every string of inputs is a fuzzy automaton automorphism identifier (i.e. h-identifier). It is established, for perfect fuzzy automaton, that the group of weak fuzzy automaton automorphisms is abelian and its cardinality is equal to the number of states of that fuzzy automaton. It is also proved that this group is a homomorphic image of the monoid of all strings of inputs. The section 4 begun by establishing the fact that, every weak fuzzy automaton isomorphism over a singly generated fuzzy automaton is determined by its value on the generator. Apart from various characterizations of existence of (weak) fuzzy automaton isomorphism over a singly generated and strongly connected fuzzy automata in terms of state fuzzy equivalence relation, various properties of weak fuzzy automaton isomorphism between two singly generated fuzzy automata are also discussed. Then the number of weak fuzzy automaton isomorphisms from a singly generated fuzzy automaton to arbitrary fuzzy automaton in terms of the maximal set of fuzzy state equivalent states is obtained. Also (weak) fuzzy automaton automorphisms over a singly generated fuzzy automaton are characterized in terms of the circular path at the generator. Finally, it is established that the number of (weak) fuzzy automaton isomorphisms over a singly generated fuzzy automaton cannot exceed the number of generators of the automaton, but it is equal to the cardinality of a maximal set of q-fuzzy automorphic set of inputs. In section 5 , it is established that any weak fuzzy automaton isomorphism on an arbitrary fuzzy automaton is completely determined by its value on the basis. Further weak fuzzy automaton isomorphism is characterized in terms of an ordered basis. Thus, we have concluded that the class of fuzzy automaton isomorphism over arbitrary fuzzy automaton can be determined with the help of weak fuzzy automaton homomorphisms on strongly connected, perfect, singly generated fuzzy automaton and primaries of fuzzy automaton 


\section{HOMOMORPHISMS AND STRONGLY CONNECTED FUZZY AUTOMATON}

The aim of this section is to introduce fuzzy automaton homomorphism and weak fuzzy automaton homomorphism in the light of the definition of fuzzy function introduced in [10]. Here, strongly connected fuzzy automaton and homomorphism on them are introduced. We measure the size of the group of (weak) fuzzy automaton isomorphisms over a strongly connected automaton.

Let $A$ and $B$ be sets. A fuzzy relation from $A$ to $B$ is a fuzzy subset $\mu$ of $A \times B$ i.e. $\mu: A \times B \rightarrow[0,1]$. The number $\mu(a, b)$ denotes the degree to which $a$ is related to $b$. A fuzzy relation $\mu$ from $A$ to $B$ is said to be complete, if for each $a \in A$, there exists $b \in B$ such that $\mu(a, b)>0$. A fuzzy relation $\mu$ is said to be fuzzy function, if for each $a \in A$, there is unique $b \in B$ such that $\mu(a, b)>0$ [10]. The above definition resembles to that of the definition of the crisp function, in the sense of unique image for each element of the domain. For preliminary notions on crisp automata theory and related concepts of this paper we refer to ([1],[6],[7],[19]).

Definition 2.1[10] A fuzzy automaton is a triplet $A=(Q, \Sigma, \mu)$, where $Q$ is a nonempty finite set called set of states, $\Sigma$ is a nonempty finite set called set of inputs and $\mu$ is a fuzzy function from $Q \times \Sigma$ to $Q$ i.e. $\mu:(Q \times \Sigma) \times Q \rightarrow[0,1]$.

The above definition of fuzzy automaton is different from the definition of $([9],[11],[12],[16])$, in the sense of defining $\mu$ only. The above definition 2.1 is, in fact, a generalization of the crisp deterministic finite state automaton, whereas definitions in $([9],[11],[12],[16])$ are generalizations of the crisp nondeterministic finite state automaton.

If $A=(Q, \Sigma, \mu)$ is a fuzzy automaton then the fuzzy function $\mu$ is extended to a fuzzy function $\mu^{*}$ from $\left(Q \times \Sigma^{*}\right)$ to $Q$ as : for all $p, q \in Q, a \in \Sigma, x \in \Sigma^{*}, \mu^{*}(p, a x, q)=\mu(p, a, r) \wedge \mu^{*}(r, x, q)$, where $r \in Q$ such that $\mu(p, a, r)>0$ and

$\mu^{*}(p, \epsilon, q)= \begin{cases}1, & \text { if } \mathrm{p}=\mathrm{q} \\ 0, & \text { otherwise }\end{cases}$

Here onwards, in this paper, we write $\mu$ for both $\mu$ and $\mu^{*}$ without any ambiguity.

Definition 2.2 Let $A_{1}=\left(Q_{1}, \Sigma_{1}, \mu_{1}\right)$ and $A_{2}=\left(Q_{2}, \Sigma_{2}, \mu_{2}\right)$ be two fuzzy automata. A a pair $(h, k)$ of maps, where $h: Q_{1} \rightarrow Q_{2}$, $k: \Sigma_{1} \rightarrow \Sigma_{2}$, is called fuzzy automaton homomorphism from $A_{1}$ to $A_{2}$, symbolically $(h, k): A_{1} \rightarrow A_{2}$, if $\forall(p, x, q) \in$ $Q_{1} \times \Sigma_{1}^{*} \times Q_{1}, \mu_{2}(h(p), k(x), h(q))=\mu_{1}(p, x, q)$.

In case, if $\Sigma_{1}=\Sigma_{2}=\Sigma$ and $k$ is the identity function on $\Sigma$, then we shall denote the homomorphism simply by $h: A_{1} \rightarrow A_{2}$. A pair of maps $(h, k): A_{1} \rightarrow A_{2}$ is said to be weak fuzzy automaton homomorphism, if $\forall(p, x, q) \in Q_{1} \times \Sigma_{1}^{*} \times Q_{1}, \mu_{1}(p, x, q)>$ $0 \Rightarrow \mu_{2}(h(p), k(x), h(q))>0$. Every fuzzy automaton homomorphism is a weak fuzzy automaton homomorphism, but not conversely. A (weak) fuzzy automaton homomorphism $(h, k)$ from $A_{1}$ to $A_{2}$ is said to be (weak) fuzzy automaton isomorphism, if both $h$ and $k$ are bijective functions.

In this paper the following notations are adopted.

$H^{F}(A \rightarrow B)$ : The set of all fuzzy automaton homomorphisms from $A$ to $B$.

$W H^{F}(A \rightarrow B)$ : The set of all weak fuzzy automaton homomorphisms from $A$ to $B$.

$I^{F}(A \rightarrow B)$ : The set of all fuzzy automaton isomorphisms from $A$ to $B$.

$W I^{F}(A \rightarrow B)$ : The set of all weak fuzzy automaton isomorphisms from $A$ to $B$.

$E^{F}(A)$ : The set of all fuzzy automaton endomorphisms on $A$.

$W E^{F}(A)$ : The set of all weak fuzzy automaton endomorphisms on $A$.
$G^{F}(A)$ : The set of all fuzzy automaton automorphisms on $A$. $W G^{F}(A)$ : The set of all weak fuzzy automaton automorphisms on $A$.

Theorem 2.3. (1) $H^{F}(A \rightarrow B) \subseteq W H^{F}(A \rightarrow B)$ (2) $I^{F}(A \rightarrow$ $B) \subseteq W I^{F}(A \rightarrow B)(3) E^{F}(A) \subseteq W E^{F}(A)$ and (4) $G^{F}(A) \subseteq$ $W G^{F}(A)$.

Lemma 2.4. Let $A=(Q, \Sigma, \mu)$ and $B=(R, \Sigma, \gamma)$ be fuzzy automata and $h \in W I^{F}(A \rightarrow B)$ then for all $(p, x, q) \in Q \times \Sigma^{*} \times Q$, we have $\gamma(h(p), x, h(q))>0 \Rightarrow \mu(p, x, q)>0$.

Proof. Let $\gamma(h(p), x, h(q))>0$. Since $\mu$ is a fuzzy function, there exists $r \in Q$ such that $\mu(p, x, r)>0$. But then by assumption $\gamma(h(p), x, h(r))>0$. This forces that $h(q)=h(r)$, as $\gamma$ is a fuzzy function. This gives us $r=q$. Therefore $\mu(p, x, q)>0$.

Theorem 2.5. If $A=(Q, \Sigma, \mu)$ is a fuzzy automaton, then $G^{F}(A)$ $\left(W G^{F}(A)\right)$ forms a group.

Definition 2.6. Let $h \in W E^{F}(A)$. An element $x_{0} \in \Sigma^{*}$ is called $h$-identifier, if $\mu\left(p, x_{0}, q\right)>0$, for $p, q \in Q$ implies that $q=h(p)$. We denote it by the symbol ' $x_{0}=h_{i d}$ '.

Remark 2.7. Note that, if $\mu$ is just a fuzzy relation from $Q \times \Sigma$ to $Q$, then $h$ can not be a function.(For this $\mu\left(p, x_{0}, q\right) \wedge \mu\left(p, x_{0}, q^{\prime}\right)>0$ gives that $h(p)=q$ as well as $h(p)=q^{\prime}$.)

The following lemma gives an important property of an $h$-identifier. Lemma 2.8. Let $A=(Q, \Sigma, \mu)$ be a fuzzy automaton, $h \in$ $W G^{F}(A)$ and $x_{0} \in \Sigma^{*}$ an $h$-identifier. Then $\mu\left(p, x_{0}^{n} y, r\right)>0$ if and only if $\mu\left(p, y x_{0}^{n}, r\right)>0, \forall y \in \Sigma^{*}$ and $\forall n \in \mathbb{N} \cup\{0\}$, where $x_{0}^{0}=\epsilon$.

Let $A=(Q, \Sigma, \mu)$ be a fuzzy automaton and $M \subseteq Q$.Then the successor of $M$ is the set $S(M)=\{p \in Q \mid \mu(q, x, p)>$ 0 , for some $\left.(q, x) \in M \times \Sigma^{*}\right\}$ and $\boldsymbol{x}$-successor of $M$ is the set $S_{x}(M)=\left\{p \in Q \mid \mu\left(q, x^{k}, p\right)>0\right.$, for some $q \in M$ and $k \in$ $\mathbb{N} \cup\{0\}\}$, where $x^{0}=\epsilon$. We denote the successor of $\{q\}$ by $S(q)$ and the $x$-successor of $\{q\}$ by $S_{x}(q)$.

Definition 2.9. A fuzzy automaton $A=(Q, \Sigma, \mu)$ is said to be strongly connected, if $q \in S(p), \forall p, q \in Q$.

The following lemma shows that two fuzzy automaton homomorphisms over strongly connected automata are identical, if they have same image for a single element.

Lemma 2.10. If $A=(Q, \Sigma, \mu)$ is a strongly connected fuzzy automaton and $h_{1}, h_{2} \in E^{F}(A)$ with $h_{1}\left(q_{0}\right)=h_{2}\left(q_{0}\right)$, for some $q_{0} \in Q$, then $h_{1}=h_{2}$.

Proof. Let $q \in Q$. Since $A$ is strongly connected fuzzy automaton, we have $q \in S\left(q_{0}\right)$.Therefore, there exists $x \in \Sigma^{*}$ such that $\mu\left(q_{0}, x, q\right)>0$. Now,

$\mu\left(h_{1}\left(q_{0}\right), x, h_{1}(q)\right)=\mu\left(q_{0}, x, q\right)$ implies that $\mu\left(h_{1}\left(q_{0}\right), x, h_{1}(q)\right)>0$. Similarly, $\mu\left(h_{2}\left(q_{0}\right), x, h_{2}(q)\right)>0$. But $h_{1}\left(q_{0}\right)=h_{2}\left(q_{0}\right)$ and $\mu$ is a fuzzy function, hence $h_{1}(q)=h_{2}(q)$.

The above lemma also holds for weak fuzzy automaton homomorphism.

Corollary 2.11. If $A=(Q, \Sigma, \mu)$ is a strongly connected fuzzy automaton and $h_{1}, h_{2} \in E^{F}(A)\left(W E^{F}(A)\right)$ with $h_{1}\left(q_{0}\right)=h_{2}\left(q_{0}\right)$, for some $q_{0} \in Q$, then $h_{1}=h_{2}$.

Theorem 2.12. If $A=(Q, \Sigma, \mu)$ is a strongly connected fuzzy automaton, then $\left|G^{F}(A)\right| \leq|Q|$.

Proof. Suppose $\left|G^{F}(A)\right|>|Q|$. Let $q_{0} \in Q$ be any fixed state. Construct $H=\left\{h\left(q_{0}\right): h \in G^{F}(A)\right\}$. Since $H \subseteq Q$, we have $\left|G^{F}(A)\right|>|H|$. Then there must exist distinct $h_{1}, \bar{h}_{2} \in$ $G^{F}(A)$ such that $h_{1}\left(q_{0}\right)=h_{2}\left(q_{0}\right)$. Thus, by Lemma 2.10, we have $h_{1}=h_{2}$. This contradicts to the fact that $h_{1}$ and $h_{2}$ are distinct. Therefore, $\left|G^{F}(A)\right| \leq|Q|$ 
Following corollary is then immediate.

Corollary 2.13. If $A=(Q, \Sigma, \mu)$ is a strongly connected fuzzy automaton, then $\left|W G^{F}(A)\right| \leq|Q|$.

\section{PERFECT FUZZY AUTOMATON}

In this section, we introduce perfect fuzzy automaton and discuss a characterization of weak fuzzy automaton homomorphism (isomorphism) in terms of $h$-identifier. This lead us to find the number of weak fuzzy automaton homomorphisms on perfect fuzzy automaton and hence number of identifiers for weak fuzzy automaton homomorphisms. Here, it is proved that the group of all weak fuzzy automaton isomorphisms on a perfect fuzzy automaton is abelian as well as a homomorphic image of monoid of strings of its inputs.

Definition 3.1. A fuzzy automaton $A=(Q, \Sigma, \mu)$ is said to be abelian, if it satisfies the condition $\mu(p, x y, q)=$ $\mu(p, y x, q), \forall x, y \in \Sigma^{*}$ and $p, q \in Q$.

If $\mu(p, x y, q)>0 \Leftrightarrow \mu(p, y x, q)>0$, for $x, y \in \Sigma^{*}$ and $p, q \in Q$, then $A$ is said to be weakly abelian

Theorem 3.2. $A=(Q, \Sigma, \mu)$ is a weakly abelian fuzzy automaton if and only if every $x_{0} \in \Sigma^{*}$ defines $h \in W E^{F}(A)$ such that $x_{0}=h_{i d}$.

Proof. Let $x_{0} \in \Sigma^{*}$. Define $h: Q \rightarrow Q$ as $h(p)=r$, where $\mu\left(p, x_{0}, r\right)>0$. Since $\mu$ is a fuzzy function, $h$ is well defined. Let $s \in Q$ and $y \in \Sigma^{*}$ such that $\mu(s, y, t)>0$ for some $t \in Q$. Now by definition of $h, \mu\left(t, x_{0}, h(t)\right)>0$. Therefore $\mu\left(s, y x_{0}, h(t)\right)>0$. Since $A$ is weakly abelian, we have $\mu\left(s, x_{0} y, h(t)\right)>0$. Thus, $\mu(h(s), y, h(t))>0$. Therefore $h$ is weak fuzzy automaton homomorphism on $A$. Clearly $x_{0}=h_{i d}$, by definition of $h$. converse holds by Lemma 2.8 .

Remark 3.3. Even though each $x_{0} \in \Sigma^{*}$ defines a weak fuzzy automaton homomorphism in the above theorem, it does not necessarily define a fuzzy automaton homomorphism whose identifier is $x_{0}$. Also, $x_{0}$ does not necessarily define weak fuzzy automaton isomorphism.

Definition 3.4. A fuzzy automaton is called perfect, if it is strongly connected and weakly abelian .

Theorem 3.5. Let $A=(Q, \Sigma, \mu)$ be a perfect fuzzy automaton. If $h \in W G^{F}(A)$ then there exists $x_{0} \in \Sigma^{*}$ such that $x_{0}=h_{i d}$. Conversely every $x_{0} \in \Sigma^{*}$, defines $h \in W G^{F}(A)$ such that $x_{0}=h_{i d}$.

Proof. Let $h \in W G^{F}(A)$ and $q_{1}, q_{2} \in Q$ be such that $h\left(q_{1}\right)=q_{2}$. Since $A$ is strongly connected, there is $x_{0} \in \Sigma^{*}$ such that $\mu\left(q_{1}, x_{0}, q_{2}\right)>0$. By Theorem 3.2 there exists $g \in$ $W E^{F}(A)$ such that $x_{0}=g_{i d}$. Since $\mu\left(q_{1}, x_{0}, q_{2}\right)>0$, we have $g\left(q_{1}\right)=q_{2}$.Then $g\left(q_{1}\right)=h\left(q_{1}\right)$, which means $g=h$ (by Lemma 2.10).Therefore $x_{0}=h_{i d}$. Conversely let $x_{0} \in \Sigma^{*}$. By Theorem 3.2, there exists $h \in W E^{F}(A)$ such that $x_{0}=h_{i d}$. Suppose $h(p)=h(s)=q$ (say). We must have $\mu\left(p, x_{0}, q\right)>$ 0 and $\mu\left(s, x_{0}, q\right)>0$. Since $A$ is strongly connected, there exists $y \in \Sigma^{*}$ such that $\mu(p, y, s)>0$.Then $\mu\left(p, y x_{0}, q\right)>0$. Hence, $\mu\left(p, x_{0} y, q\right)>0$, as $A$ is perfect. This gives us $\mu\left(p, x_{0}, q\right) \wedge$ $\mu(q, y, q)>0$. Therefore $\mu(q, y, q)>0$. Then again there is $f \in$ $W E^{F}(A)$ such that $f_{i d}=y$. Therefore, $f(q)=q$. Then $f$ must be identity function on $A$ (due to Lemma 2.10). Hence $f(p)=p$. But $\mu(p, y, s)>0$ thus $f(p)=s$. This gives us $p=s$. Therefore $h$ is one - one. Also $h$ is onto, as $Q$ is finite. Hence $h \in W G^{F}(A)$.

The abelian property of the $W G^{F}(A)$ is an easy consequence of the above theorem.

Corollary 3.6. If $A=(Q, \Sigma, \mu)$ is a perfect fuzzy automaton, then $W G^{F}(A)$ is abelian.
The following corollary is establishing our goal of finding the number of weak fuzzy automaton homomorphisms on perfect fuzzy automaton.

Corollary 3.7. If $A=(Q, \Sigma, \mu)$ is a perfect fuzzy automaton, then $\left|W G^{F}(\AA)\right|=|Q|$.

Proof. Let $q_{0} \in Q$. Define $f: Q \rightarrow W G^{F}(A)$ by $f(q)=h$, where $h_{i d}=x$ and $\mu\left(q_{0}, x, q\right)>0$. We first prove that $f$ is well defined. Let $q_{1}=q_{2}$ with $\mu\left(q_{0}, x, q_{1}\right)>0$ and $\mu\left(q_{0}, y, q_{2}\right)>0$. Let $h_{i d}=x$ and $g_{i d}=y$. Then $f\left(q_{1}\right)=h$ and $f\left(q_{2}\right)=g$. Since $\mu\left(q_{0}, x, q_{1}\right)>0$, we have $\mu\left(g\left(q_{0}\right), x, g\left(q_{1}\right)\right)>0$. Thus, by definition of $h, h\left(g\left(q_{0}\right)\right)=g\left(q_{1}\right)$. Similarly, $g h\left(q_{0}\right)=h\left(q_{2}\right)$. But then by Corollary 3.6, one has $h g=g h$. Therefore, $g\left(q_{1}\right)=h\left(q_{2}\right)$. This gives us $g=h$, as $q_{1}=q_{2}$. Thus $f\left(q_{1}\right)=f\left(q_{2}\right)$. Hence, $f$ is well defined. The one-oneness of $f$ will imply that $|Q| \leq\left|W G^{F}(A)\right|$. Thus we prove $f$ is $1-1$. Let $f\left(q_{1}\right)=f\left(q_{2}\right)$. Then $\mu\left(q_{0}, x, q_{1}\right)>0$ and $\mu\left(q_{0}, x, q_{2}\right)>0$, gives $q_{1}=q_{2}$. Clearly Corollary 2.13 proves the other inequality and hence the theorem.

The following theorem establishes the relationship between $W G^{F}(A)$ and $\Sigma^{*}$ as monoids.

Theorem 3.8. Let $A=(Q, \Sigma, \mu)$ be a perfect fuzzy automaton. Then $W G^{F}(A)$ is a homomorphic image of $\Sigma^{*}$.

Proof. Define $\phi: \Sigma^{*} \rightarrow W G^{F}(A)$ by $\phi(x)=h$, if $h_{i d}=x$. Let $\phi(x)=h, \phi(y)=k$ and $\phi(x y)=g$. Then $g(q)=t_{1}$, whenever $\mu\left(q, x y, t_{1}\right)>0$. Since $A$ is weakly abelian, we have $\mu\left(q, y x, t_{1}\right)>0$. Thus, $\mu(q, y, t)>0$ and $\mu\left(t, x, t_{1}\right)>0$, for some $t \in Q$. Therefore, $k(q)=t$ and $h(t)=t_{1}$. This gives $h(k(q))=t_{1}=g(q)$. Thus, $h k=g$. Therefore, $\phi(x y)=g=$ $h k=\phi(x) \phi(y)$. Hence, $W G^{F}(A)$ is a homomorphic image of $\Sigma^{*}$.

\section{SINGLY GENERATED FUZZY AUTOMATON}

The sole aim of this section is to identify weak fuzzy automaton homomorphisms over singly generated automaton, over primaries and over any fuzzy automata. Thus in this section we study singly generated fuzzy automaton. Through primary decomposition theorem, we show that every arbitrary finite fuzzy automaton can be decomposed into its major substructures - i.e. its primaries. Therefore we can discuss weak fuzzy automaton homomorphism over arbitrary fuzzy automaton in the next section via primaries. In the present section we concentrate to find group of weak fuzzy automaton isomorphisms over singly generated fuzzy automata.

Let $A=(Q, \Sigma, \mu)$ be a fuzzy automaton and $q \in Q$. A fuzzy automaton generated by $q$ is denoted by $A(q)$ and is a triplet $A(q)=$ $\left(S(q), \Sigma, \mu^{\prime}\right)$, where $\mu^{\prime}$ is a restriction of $\mu$ to $S(q) \times \Sigma \times S(q)$. A fuzzy automaton A is said to be singly generated, if there exists $q \in Q$ such that $A=A(q)$. The set of generators of $A(q)$ is the set $\operatorname{gen} A(q)=\left\{r \in S_{A(q)} \mid A(r)=A(q)\right\}$.

Every $f \in W E^{F}(A(q))$ is completely determined by its value on $q$, in the sense that the value $f(p)$ for any element $p \in Q$ is determined by the value of $f(q)$.

Lemma 4.1. Every $f \in W E^{F}(A(q))$ is completely determined by its value on $q$.

Proof. Let $t \in Q$. Then there exists $x \in \Sigma^{*}$ such that $\mu(q, x, t)>0$. Now, $\mu(f(q), x, f(t))>0$ gives $f(t)=s$, if $\mu(f(q), x, s)>0$. This shows that $f$ is completely determined by its value on $q$.

The following corollary is an improvement over Lemma 2.10 for finding an element $q_{0}$ as its generator.

Corollary 4.2. Let $f, g \in W E^{F}(A(q))$. Then $f=g$ if and only if 
$f(q)=g(q)$.

By taking $g$ as the identity function, we have

Corollary 4.3. $f \in W E^{F}(A(q))$ is the identity if and only if it fixes a generator of $A(q)$; i.e. $f(q)=q \Leftrightarrow \forall r \in Q, f(r)=r$.

Recall that $B=\left(Q^{\prime}, \Sigma, \mu^{\prime}\right)$ is a subautomaton of $A=(Q, \Sigma, \mu)$, if $Q^{\prime} \subseteq Q, S\left(Q^{\prime}\right)=Q^{\prime}$ and $\mu^{\prime}=\left.\mu\right|_{Q^{\prime} \times \Sigma \times Q^{\prime}}$ [18]. We shall denote it by $B \ll A$.

Definition 4.4. A subautomaton $B$ of $A$ is called a primary of $A$, if (i) there exists $q \in Q$ such that $B=A(q)$ and

(ii) for any $t \in Q$, if $B \ll A(t)$, then $B=A(t)$.

Definition 4.5. Let $A=(Q, \Sigma, \mu)$ be a fuzzy automaton and $P_{1}=\left(Q_{1}, \Sigma, \mu_{1}\right), P_{2}=\left(Q_{2}, \Sigma, \mu_{2}\right)$ are primaries of $A$. Then define $P_{1} \cup P_{2}$ as a subautomaton $\left(Q_{1} \cup Q_{2}, \Sigma, \mu_{1} \vee \mu_{2}\right)$ of $A$. In case of two distinct singly generated subautomata of a given fuzzy automaton, the generator of one of them may belongs to the other. However, we have

Theorem 4.6. Let $A=(Q, \Sigma, \mu)$ be a fuzzy automaton. Two distinct primaries of $A$ does not have any generator in common.

The following theorem shows that the primaries are the important substructures of an arbitrary finite fuzzy automaton. Hence, we call this theorem as primary decomposition theorem.

Theorem 4.7. If $A=(Q, \Sigma, \mu)$ is a fuzzy automaton and $\pi=$ $\left\{P_{1}, P_{2}, \ldots, P_{n}\right\}$ is the set of distinct primaries of $A$, where $P_{i}=$ $\left(Q_{i}, \Sigma, \mu_{i}\right), Q_{i} \subseteq Q, \mu_{i}$ is the restriction of $\mu$ to $Q_{i} \times \Sigma \times Q_{i}, \forall i$ , then

(i) $A=\bigcup_{i=1}^{n} P_{i}$. (ii) for any $j \in\{1,2, \ldots, n\}, A \neq \bigcup_{j \neq i=1}^{n} P_{i}$.

Proof. (i) Let $q_{0} \in Q$. If $A\left(q_{0}\right)$ is not a primary of $A$, then there is $q \in Q-S\left(q_{0}\right)$ such that $A\left(q_{0}\right) \ll A(q)$ and $A(q) \in \pi$. Hence $Q=\bigcup_{i=1}^{n} Q_{i}$. Also, since each $\mu_{i}$ is a restriction of $\mu$, we have $\mu=\bigvee_{i=1}^{n} \mu_{i}$. Therefore $A=\bigcup_{i=1}^{n} P_{i}$.

(ii) Let $B=\bigcup_{j \neq i=1}^{n} P_{i}$ and let $P_{j}=A(q)$, for some $j \in$ $\{1,2, \ldots, n\}$. If $q \in Q_{B}$ (the state set of B), then $q \in Q_{i}$ for some $i \neq j$ and $P_{j}=A(q) \ll P_{i}$ (by (i)). This contradicts the maximality of $P_{j}$, since $P_{i} \neq P_{j}$. Therefore, $q$ does not belong to $Q_{B}$ and hence $A \neq \bigcup_{j \neq i=1}^{n} P_{i}$.

Remark 4.8. Tiwari and Srivastava [18] have also introduced primaries and primary decomposition theorem for compact fuzzy automaton. The approach in [18] was purely topological whereas our approach is purely algebraic. In the present paper fuzzy automaton is finite and is based on fuzzy function, this makes our approach different than that in [18].

The following lemma shows that a singly generated subautomaton of a fuzzy automaton (or a primary) is preserved under weak fuzzy automaton homomorphism (or isomorphism).

Lemma 4.9. Let $A=(Q, \Sigma, \mu)$ and $B=(R, \Sigma, \gamma)$ be fuzzy automata and $f \in H^{F}(A \rightarrow B), h \in I^{F}(A \rightarrow B)$ and $P \ll A$. Then

(i) $\forall q \in Q, f[A(q)]=A[f(q)]$.

(ii) for any $t \in Q$, we have $h(S(t))=S(h(t))$.

(iii) $P$ is a primary of $A$ if and only if $h(P)$ is a primary of $\mathrm{B}$.

$$
\begin{aligned}
& \text { Proof. (i) } f\left(S\left(q_{0}\right)\right)=f\left[\bigcup_{x \in \Sigma^{*}}\left\{q \in Q \mid \mu\left(q_{0}, x, q\right)>0\right\}\right] \\
= & \bigcup_{x \in \Sigma^{*}}\left\{f(q) \in Q \mid \gamma\left(f\left(q_{0}\right), x, f(q)\right)>0\right\} \text { (since } f \text { is fuzzy au- }
\end{aligned}
$$

tomaton homomorphism. $)=\bigcup_{x \in \Sigma^{*}}\left\{t \in R \mid \gamma\left(f\left(q_{0}\right), x, t\right)>0\right\}$ (since $\gamma$ is a fuzzy function.) $=\gamma\left(f\left(q_{0}\right)\right)$. Therefore, $f[A(q)]=$ $A[f(q)]$.

(ii) Proof of this is obvious due to the condition that for $h \in$ $I^{F}(A \rightarrow B), \mu(t, x, p)>0$ if and only if $\gamma(h(t), x, h(p))>0$. (iii) Let $P$ be any primary of $A$. Then $A(q)=P$, for some $q \in Q$. Therefore, by (i), $h(P)=h[A(q)]=A[h(q)]$. Hence, by Theorem 4.7, we have $h(q)$ is a state of some primary $A(h(t))$ of $B$ and $h(q) \in S(h(t))$. Since $h^{-1} \in I^{F}(B \rightarrow A)$,we have $q=h^{-1}(h(q)) \in h^{-1}(S(h(t)))=S(t)$. This implies that $A(q) \ll A(t) \Rightarrow A(q)=A(t)$, since $A(q)$ is a primary of $A$, thus, by (i), $A[h(q)]=A[h(t)]$. Therefore $A[h(q)]$ is a primary of $B$. The converse of (iii) can be proved similarly.

Definition 4.10. Let $A=(Q, \Sigma, \mu)$ be a fuzzy automaton and $q \in Q, y, z \in \Sigma^{*}$. Then $y$ is said to be $q$-fuzzy equivalent to $z$, if $\mu(q, y, p)>0$ and $\mu(q, z, p)>0$, for some $p \in Q$. We shall denote it by $y \equiv_{q}^{F} z$.

Remark 4.11. $\equiv_{q}^{F}$ is an equivalence relation of finite index.

The following theorem characterize the weak fuzzy automaton homomorphism (and isomorphism) in terms of above state-fuzzy equivalence relation.

Theorem 4.12. Let $A=A(q)$ and $B=(R, \Sigma, \gamma)$ be fuzzy automata. Then

(i) there exists $f \in W H^{F}(A(q) \rightarrow B)$ if and only if $\exists t \in R$ satisfying $\forall y, z \in \Sigma^{*}, y \equiv_{q}^{F} z \Rightarrow y \equiv_{t}^{F} z$.

(ii) there exists $f \in W I^{F}(A(q) \rightarrow B)$ if and only if $\exists t \in \operatorname{gen}(B)$ satisfying $\forall y, z \in \Sigma^{*}, y \equiv_{q}^{F} z \Leftrightarrow y \equiv_{t}^{F} z$.

Moreover, $f$ is completely determined by its value on $q$ as follows: $f(q)=t$ and for all $p \in A(q), p \neq q$ with $\mu(q, w, p)>0$, for some $w \in \Sigma^{*}$, we have $f(p)=r$, where $\gamma(t, w, r)>0$.

Proof. (i) Suppose there exists $t \in R$ such that $\forall y, z \in$ $\Sigma^{*}, y \equiv_{q}^{F} z \Rightarrow y \equiv_{t}^{F} z$. Define $f: A(q) \rightarrow B$ by $f(p)=r$ , whenever $\mu(q, w, p)>0$ and $\gamma(t, w, r)>0$, for some $w \in \Sigma^{*}$. Let $a=b$ and $\mu(q, y, a)>0, \mu(q, z, b)>0$. Therefore $y \equiv_{q}^{F} z$. Hence $y \equiv_{t}^{F} z$. Then $\gamma(t, y, s)>0$ and $\gamma(t, z, s)>0$ for some $s \in R$, which gives $f(a)=s=f(b)$. Therefore, $f$ is well defined. Since $\mu(q, \epsilon, q)>0$, we have $f(q)=t$ (as $\gamma(t, \epsilon, t)>0$ ). Suppose $p, r \in Q$ be such that $\mu(p, x, r)>0$, for $x \in \Sigma^{*}$. Let $u \in \Sigma^{*}$ be such that $\mu(q, u, p)>0$. Then $\mu(q, u x, r)>0$. By definition of $f$, $f(r)=s$ whenever $\gamma(t, u x, s)>0$. Hence, $\gamma(t, u, f(p))>0$ and $\gamma(f(p), x, s)>0$.

Thus, we conclude that whenever $\mu(p, x, r)>0$, then $\gamma(f(p), x, f(r))>0$. This proves that $f$ is a weak fuzzy automaton homomorphism. Conversely, let $f$ be a weak fuzzy automaton homomorphism. Then by taking $f(q)=t$, one proves the result. Similarly one can prove (ii).

Note that $\equiv_{q}^{F}$ is not a congruence relation. But

Corollary 4.13. There exists $f \in W E^{F}(A(q))$ if and only if there exists $x \in \Sigma^{*}$ satisfying $\forall y, z \in \Sigma^{*}, y \equiv_{q}^{F} z \Rightarrow x y \equiv_{q}^{F} x z$.

Moreover, $f$ is completely determined by its value on $q$ as follows $: f(q)=t$, if $\mu(q, x, t)>0$ and for any $p \in Q, p \neq q$, we have $f(p)=r$, if $\mu(q, w, p)>0$ and $\mu(q, x w, r)>0, \forall w \in \Sigma^{*}$.

Corollary 4.14. There exists $f \in W G^{F}(A(q))$ if and only if there exists $x \in \Sigma^{*}$ satisfying $\forall y, z \in \Sigma^{*}, y \equiv_{q}^{F} z \Leftrightarrow x y \equiv_{q}^{F} x z$.

Corollary 4.15. If $A$ is a strongly connected fuzzy automaton, then there is $f \in W E^{F}(A)$ if and only if there exists $x \in \Sigma^{*}$ satisfying $\forall y, z \in \Sigma^{*}, y \equiv_{q}^{F} z \Rightarrow x y \equiv_{q}^{F} x z, \forall q \in Q$.

In the following theorem we use symbols $f(S)$ and $S(f)$ for $f \circ g$ and $g \circ f$ respectively, for each $g \in S$. (Note that $S$ is set of functions such that $f \circ g$ and $g o f$ are defined). 
Theorem 4.16. If $W I^{F}[A(q) \rightarrow A(t)] \neq \phi$ then (i) $W I^{F}[A(q) \rightarrow A(t)]=f\left(W G^{F}(A(q))\right)=\left(W G^{F}(A(t))\right) f$, $\forall f \in W I^{F}[A(q) \rightarrow A(t)]$

(ii) $\left|W I^{F}[A(q) \rightarrow A(t)]\right|=\left|\left(W G^{F}(A(q))\right)\right|=$ $\left|\left(W G^{F}(A(t))\right)\right|$.

Proof. Let $f \in W I^{F}(A(q) \rightarrow A(t))$. For any $g \in W G^{F}(A(q)), f g \quad \in W I^{F}(A(q) \rightarrow A(t))$. Therefore $f\left(W G^{F}(A(q))\right) \subseteq W I^{F}(A(q) \rightarrow \rightarrow A(t))$. Also for any $h \in W I^{F}(A(q) \rightarrow A(t))$, we have $f^{-1} h \in W G^{F}(A(q))$. Then $h \in f\left(W G^{F}(A(q))\right)$. Therefore, $W I^{F}(A(q) \rightarrow A(t)) \subseteq f\left(W G^{F}(A(q))\right)$. Similarly $W I^{F}[A(q) \rightarrow A(t)] \stackrel{=}{=}\left(W G^{F}(A(t))\right) f$. This proves $W I^{F}[A(q) \rightarrow A(t)]=f\left(W G^{F}(A(q))\right)=$ $\left(W G^{F}(A(t))\right) f, \forall f \in W I^{F}[A(q) \rightarrow A(t)]$. Since $f$ is one-one, we have $f g_{1}=f g_{2} \Leftrightarrow g_{1}=g_{2}, \forall g_{1}, g_{2} \in W G^{F}(A(q))$, follows $\left|W I^{F}[A(q) \quad \rightarrow \quad A(t)]\right| \stackrel{=}{\rightarrow}\left|\left(W G^{F}(A(q))\right)\right|=$ $\left|\left(W G^{F}(A(t))\right)\right|$.

Theorem 4.17. Let $A=\left(Q, \Sigma, \mu_{1}\right)$ and $B=\left(R, \Sigma, \mu_{2}\right)$.

(i) Let $q \in Q$ be a fixed state and let $P$ be a maximal set of states of $B$ such that for each $p$ of $P$, we have $y \equiv_{q}^{F} z \Rightarrow y \equiv_{p}^{F} z, \forall y, z \in$ $\Sigma^{*}$. Then $W H^{F}[A(q) \rightarrow B]=\left\{f_{p}: p \in P\right\}$, where $f_{p}(s)=t$, whenever $\mu(q, u, s)>0$ and $\gamma(p, u, t)>0$,for some $u \in \Sigma^{*}$. Moreover, $\left|W H^{F}[A(q) \rightarrow B]\right|=|P|$.

(ii) Let $T$ be a maximal set of states of $B$ such that for each $t$ of $T$ we have $y \equiv_{q}^{F} z \Leftrightarrow y \equiv_{t}^{F} z, \forall y, z \in \Sigma^{*}$. Then the set of all weak fuzzy automaton monomorphisms on $A(q)$ to $B$ is the set $\left\{f_{t}: t \in\right.$ $T\}$. If $B$ is a singly generated, then $W I^{F}[A(q) \rightarrow B]=\left\{f_{t}: t \in\right.$ $T \bigcap$ gen $B\}$. Moreover, $\left|W I^{F}[A(q) \rightarrow B]\right|=\mid T \bigcap$ gen $B \mid$.

Proof. (i) Let $p \in P$. Then for any $y, z \in \Sigma^{*}, y \equiv_{q}^{F}$ $z \Rightarrow y \equiv{ }_{p}^{F} z$. Hence by Theorem 4.12, there exists $f_{p} \in$ $W H^{F}(A(q) \rightarrow B)$ such that $f_{p}(q)=p$.

Conversely, let $f \in W H^{F}(A(q) \rightarrow B)$. Then there exists $p \in R$ such that $\forall y, z \in \Sigma^{*}, y \equiv_{q}^{F} z \Rightarrow y \equiv_{p}^{F} \quad z$.(By Theorem 4.12). Hence, $p \in P$. Thus, there is one to one correspondence between $W H^{F}[A(q) \rightarrow B]$ and $\left\{f_{p}: p \in P\right\}$ and $\left\{f_{p}: p \in P\right\} \subseteq$ $W H^{F}[A(q) \rightarrow B]$. Therefore, $W H^{F}[A(q) \rightarrow B]=\left\{f_{p}: p \bar{\epsilon}\right.$ $P\}$. Since $P$ is maximal, we must have $\left|W H^{F}[A(q) \rightarrow B]\right|=$ $\left|\left\{f_{p}: p \in P\right\}\right|$. Similarly one can prove (ii).

Definition 4.18. Let $A=(Q, \Sigma, \mu)$ be any fuzzy automaton and $q \in Q, x \in \Sigma^{*}$. Then the $\boldsymbol{x}$-path of $q$ is the subautomaton $O_{x}(q)=\left(S_{x}(q),\{x\}, \mu^{\prime}\right)$, where $\mu^{\prime}$ is the restriction of $\mu$ to $S_{x}(q) \times\{x\} \times S_{x}(q)$. The $\boldsymbol{x}$ circle of $\boldsymbol{q}$ is the subautomaton $C_{x}(q)=\left(S_{x}^{c}(q),\{x\}, \mu^{\prime}\right)$, where $S_{x}^{c}(q)=\left\{t \in S_{x}(q)\right.$ : $\mu\left(q, x^{k}, t\right)>0$ and $\mu\left(q, x^{m}, t\right)>0$, for some integer $\left.m>k\right\}$ and $\mu^{\prime}$ is the restriction of $\mu$ to $S_{x}^{c}(q) \times\{x\} \times S_{x}^{c}(q)$. The $x$ - path of $q, O_{x}(q)$ is said to be circular, if $O_{x}(q)=C_{x}(q)$.

Note : For any $x \in \Sigma^{*}$, we have $A(x)=\bigcup_{q \in Q} O_{x}(q)$.

Definition 4.19. Let $A=(Q, \Sigma, \mu)$ and $f: Q \rightarrow Q$.

Then the $\boldsymbol{f}$-path of $q \in Q$ is the set $O_{f}(q)=\left\{f^{k}(q)\right.$ for some $k \in$ $\mathbb{N} \cup\{0\}\}$, where $f^{0}(q)=q$. The $\boldsymbol{f}$ circle of $q$ is the set $C_{f}(q)=$ $\left\{f^{k}(q) \in O_{f}(q): f^{k}(q)=f^{m}(q)\right.$, for some integer $\left.m>k\right\}$.

The $f$ - path of $q, O_{f}(q)$ is said to be circular, if $O_{f}(q)=C_{f}(q)$.

Lemma 4.20. (1) For some $r \in Q, r \in C_{f}(r)$ if and only if $O_{f}(r)=C_{f}(r)$.

(2) $O_{f}(r)=\{r\}$ if and only if $f(r)=r$.

(3) For some $x \in \Sigma^{*}$ and $h \in W G^{F}(A)$, if $h_{i d}=x$ with $o(h)=k$, then $O_{x}(q)=C_{x}(q)$.

(4) $x \in \Sigma^{*}, q \in Q$. If $O_{x}(q)=C_{x}(q)$ and $t \in S_{x}(q)$, then $O_{x}(t)=C_{x}(t)$.
Theorem 4.21 Let $A=(Q, \Sigma, \mu)$ be a fuzzy automaton and let $h \in W G^{F}(A)$. Then $O_{h}(r)$ is circular for any $r \in Q$. If $A=A(q)$ , then there exists $x \in \Sigma^{*}$ such that $O_{x}(q)$ is circular.

Proof. Since $W G^{F}(A)$ is a finite group, we have the order of $h$ is finite. Thus, for any $r \in Q, h^{O(h)}(r)=r$. Therefore, $r \in C_{h}(r)$. Then $C_{h}(r)=O_{h}(r)$. i.e. $O_{h}(r)$ is circular. Let $A=A(q)$. Then by Corollary 4.13 , there exists $x \in \Sigma^{*}$ such that $h(q)=t_{1}$, whenever $\mu\left(q, x, t_{1}\right)>0$, for some $t_{1} \in Q$.If $\mu\left(q, x^{2}, t_{2}\right)>0$ for some $t_{2} \in Q$, then $h^{2}(q)=h(h(q))=$ $h\left(t_{1}\right)=t_{2}$. In general for any $n \in \mathbb{N}$, if $\mu\left(q, x^{n}, t_{n}\right)>0$, for some $t_{n} \in Q$, then $h^{n}(q)=t_{n}$. In particular for $n=k$ (the order of $h$ ), we have $t_{k}=q$. Then $\mu\left(q, x^{k}, q\right)>0$. Hence, $O_{x}(q)=C_{x}(q)$.

Theorem 4.22. There exists $f \in W G^{F}(A(q))$ such that $f \neq i d$ if and only if there exists $x \in \Sigma^{*}$ satisfying :

(i) $\mu(q, x, q)=0$ (ii) $O_{x}(q)=C_{x}(q)$ and (iii) $\forall y, z \in \Sigma^{*}, y \equiv_{q}^{F}$ $z \Rightarrow x y \equiv_{q}^{F} x z$.

Further, for each $u \in \Sigma^{*}$, if $x \equiv_{q}^{F} u$, then $O_{u}(q)=C_{u}(q)$.

Proof. Let $f \in W G^{F}(A(q))$ such that $f \neq i d$. Then by Corollary 4.14 there exists $x \in \Sigma^{*}$ such that $f(q)=t$, whenever $\mu(q, x, t)>0$. Satisfying, (i) and (iii) and by Theorem 4.21, (ii) also holds . Conversely suppose there exists $x \in \Sigma^{*}$ satisfying the conditions. Define $f_{x}(p)=s$, whenever $\mu(q, w, p)>0$ and $\mu(q, x w, s)>0$, for some $w \in \Sigma^{*}$. Then by Corollary 4.13 , $f_{x}$ is a non-identity weak fuzzy automaton homomorphism. Since $O_{x}(q)$ is circular, $\mu\left(q, x^{n}, q\right)>0$, for some $n \in \mathbb{N}$. Now, for any $r \in Q$ with $\mu(q, w, r)>0$, let $\mu\left(q, x w, r_{1}\right)>0$ for some $r_{1} \in Q$, then $f(r)=r_{1}$. Let $\mu\left(q, x^{2} w, r_{2}\right)>0$ for some $r_{2} \in Q$, then $f^{2}(r)=f(f(r))=f\left(r_{1}\right)=r_{2}$. Similarly $\mu\left(q, x^{3} w, r_{3}\right)>0$ for some $r_{3} \in Q$, then $f^{3}(r)=f\left(f^{2}(r)\right)=f\left(r_{2}\right)=r_{3}$. In general $f^{n}(r)=r_{n}$, whenever $\mu\left(q, x^{n} w, r_{n}\right)>0$ for some $r_{n} \in Q$. This implies that $\mu\left(q, w, r_{n}\right)>0$. Since $\mu$ is a fuzzy function, we have $r_{n}=r$. Thus, $f^{n}(r)=r$. i.e. $f\left(f^{n-1}(r)\right)=r$. Therefore, $f$ is onto. Finiteness of $Q$ implies that $f$ is one one also. Thus, $f \in W G^{F}(A(q))$. Next $x \equiv_{q}^{F} u$ implies that $\mu(q, x, p)>0$ and $\mu(q, u, p)>0$ for some $p \in Q$. Then by condition (iii) $x^{2} \equiv_{q}^{F} x u$. Therefore $\mu\left(q, x^{2}, r\right)>0$ and $\mu(q, x u, r)>0$, for some $r \in Q$. Then $\mu(p, u, r)>0$. Thus $\mu\left(q, x^{2}, r\right)>0$ and $\mu\left(q, u^{2}, r\right)>0$. i.e. $x^{2} \equiv_{q}^{F} u^{2}$. In general,for any $n \in \mathbb{N}$, we have $x^{n} \equiv_{q}^{F} u^{n}$. This proves that $O_{u}(q)=C_{u}(q)$.

Corollary 4.23. There exists $f \in W G^{F}(A(q))$ such that $f \neq i d$ if and only if there exists $x \in \Sigma^{*}$ satisfying :

(i) $O_{x}(q)=C_{x}(q)$ (ii) $\forall y, z \in \Sigma^{*}, y \equiv_{q}^{F} z \Rightarrow x y \equiv_{q}^{F} x z$.

Theorem 4.24. Let $f_{x} \in W E^{F}(A(q)), x \in \Sigma^{*}$ be such that $f_{x}(q)=t$, whenever $\mu(q, x, t)>0$.Then $O_{x}(q)=C_{x}(q)$ if and only if $t \in$ gen $A(q)$.

Proof. Suppose $O_{x}(q)=C_{x}(q)$. Then $\mu\left(q, x^{n}, q\right)>0$, for some positive integer $n$. But then, $\mu\left(t, x^{n-1}, q\right)>0$, since $\mu(q, x, t)>0$. This implies that $t \in \operatorname{gen} A(q)$. Conversely suppose that $t \in \operatorname{gen} A(q)$ i.e. $f_{x}(q) \in \operatorname{gen} A(q)$. Then $A\left[f_{x}(q)\right]=A(q)$. Thus by Lemma 4.9, $f_{x}[A(q)]=A(q)$. Therefore, $f_{x}$ is onto. Also, $f_{x}$ is one one, as $Q$ is finite. Thus, $f_{x} \in W G^{F}(A(q))$. Hence, by Theorem 4.22, $O_{x}(q)=C_{x}(q)$.

Corollary 4.25. Let $f \in W E^{F}(A(q))$. Then the following are equivalent

(i) $f \in W G^{F}(A(q))$.

(ii) $f(q) \in$ gen $A(q)$.

(iii) $O_{f}(q)=C_{f}(q)$. 
Definition 4.26. Let $A=(Q, \Sigma, \mu)$, let $q \in Q$, and $H$ be a subset of $W G^{F}(A)$. Then $H$ image of $q$ is $I_{H}(q)=\{h(q): h \in H\}$. If $H=W G^{F}(A)$, then we shall denote $H$ image of $q$ by $I_{G}(q)$. Note that $I_{\{f\}}(q) \subseteq O_{f}(q)$.

These $H$ images for different states, in case of strongly connected fuzzy automaton, forms a partition of $Q$. This fact follows due to Lemma 2.10 .

Lemma 4.27. Let $A=(Q, \Sigma, \mu)$ be strongly connected fuzzy automaton. Let $p, q \in Q$, and $H$ be a subset of $W G^{F}(A)$. Then $I_{H}(p)$ and $I_{H}(q)$ are either disjoint or identical .

Corollary 4.28. For any $q \in Q,\left|I_{H}(q)\right|=|H|$.

Theorem 4.29. Let $A$ be a singly generated fuzzy automaton with generator $q$. Then $\left|W G^{F}(A(q))\right|$ divides $|\operatorname{gen} A(q)|$.

Proof. Let $t \in \operatorname{gen} A(q)$. Due to the Corollary 4.26(ii), $I_{G}(t) \subseteq \operatorname{gen} A(q)$. If $t_{1}, t_{2} \in \operatorname{gen} A(q)$, then $I_{G}\left(t_{1}\right)$ and $I_{G}\left(t_{2}\right)$ are either identical or disjoint (by Lemma 4.27). Since $t \in I_{G}(t)$ for every $t$, we have $\bigcup_{t \in g e n} I_{G}(t)=\operatorname{gen} A(q)$. Thus, $W G^{F}(A(q))$ partitions gen $A(q)$ into disjoint subsets of the form $I_{G}(t)$ with $t \in \operatorname{gen} A(q)$. Also due to the Corollary 4.28, we have $\left|I_{G}(t)\right|=$ $\left|W G^{F}(A(q))\right|$, for every $t \in \operatorname{gen} A(q)$. Therefore $|\operatorname{gen} A(q)|=$ $n\left|W G^{F}(A(q))\right|$, where $n$ is the number of distinct paths of $\operatorname{gen}(A(q))$. Hence, $\left|W G^{F}(A(q))\right|$ divides $|\operatorname{gen} A(q)|$.

Corollary 4.30. If $g \in W G^{F}(A(q))$, then $\left|O_{g}(q)\right|$ divides $|\operatorname{gen} A(q)|$

Corollary 4.31. For any $r \in Q$ and $g \in W G^{F}(A(q)),\left|O_{g}(r)\right|$ divides $\left|O_{g}(q)\right|$.

The Corollary 4.13(and Corollary 4.14 ) motivates us to collect $x \in \Sigma^{*}$ to determine total number of elements of $W E^{F}(A)$ (and $W G^{F}(A)$ ). Precisely the following definition fulfill our goal (See Theorem 4.33).

Definition 4.32. Let $A=(Q, \Sigma, \mu)$ be a fuzzy automaton and $q \in Q$. A subset $X_{q}$ of $\Sigma$ is $q$-fuzzy endomorphic, if (i) For all $x, w \in X_{q}$,if $x \equiv_{q}^{F} w$ then $x=w$. and (ii) For $x \in X_{q}$ and $y, z \in \Sigma^{*}$, if $y \equiv_{q}^{F} z$ then $x y \equiv_{q}^{F} x z$.

Also if $O_{x}(q)=C_{x}(q), \forall x \in X_{q}$ then $X_{q}$ is called $q$-fuzzy automorphic .

Theorem 4.33. Let $X_{q}$ be a maximal q-fuzzy endomorphic set of inputs. Then $W E^{F}(A(q))=\left\{f_{x}: x \in X_{q}\right\}$, where $f_{x}(s)=t$, if $\mu(q, u, s)>0$ and $\mu(q, x u, t)>0, \forall u \in \Sigma^{*}$. Moreover, $\left|W E^{F}(A(q))\right|=\left|X_{q}\right|$.

If $X_{q}$ is a maximal q-fuzzy automorphic set of inputs. Then $W G^{F}(A(q))=\left\{f_{x}: x \in X_{q}\right\}$. Moreover, $\left|W G^{F}(A(q))\right|=$ $\left|X_{q}\right|$.

Proof. Let $x \in X_{q}$. Then by Corollary 4.13, the mapping $f_{x}(q)=p$, whenever $\mu(q, x, p)>0$ defines weak fuzzy automaton endomorphism $f_{x}$ on $A(q)$.

On the other hand, if $f \in W E^{F}(A(q))$, then by corollary 4.13 , there exists $x \in \Sigma^{*}$ such that $f=f_{x}$. Therefore $W E^{F}(A(q))=$ $\left\{f_{x}: x \in X_{q}\right\}$. By maximality of $X_{q}$ we have $\left|W E^{F}(A(q))\right|=$ $\left|X_{q}\right|$. Similarly by Corollary 4.14 ,if $X_{q}$ is a maximal q-fuzzy automorphic set of inputs, then $W G^{F}(A(q))=\left\{f_{x}: x \in X_{q}\right\}$ and $\left|W G^{F}(A(q))\right|=\left|X_{q}\right|$.

\section{WEAK FUZZY AUTOMATON ISOMORPHISMS ON ARBITRARY FUZZY AUTOMATA}

As it is pointed out earlier, this section is focused on characterization of weak fuzzy automaton isomorphism over arbitrary automata through primaries, basis, generating set and ordered basis.

Definition 5.1. A subset $R$ of $Q$ is a generating set of a fuzzy au- tomaton $A=(Q, \Sigma, \mu)$, if $A=\bigcup_{r \in R} A(r)$.

A minimal generating set of $A$ is called a basis of $A$. Due to the primary decomposition theorem 4.7 the following are obvious.

Theorem 5.2. Every fuzzy automaton has a basis.

Proof. The set $R$ consisting of only one generator from each primary of $A$ is a basis.

Theorem 5.3. Any two bases of a fuzzy automaton $A$ has same number of elements.

The number of elements in a basis is called the rank of $A$. Therefore rank of $A$ is the number of distinct primaries of $A$.

The restriction of a weak fuzzy automaton homomorphism of $A$ to a primary $P$ is a weak fuzzy automaton homomorphism of $P$. This homomorphism is completely determined by its value on any generator of $P$.(by Lemma 4.1) and by primary decomposition theorem, each state of $A$ is a state of some primary $P$ of $A$. Therefore a weak fuzzy automaton homomorphism on fuzzy automata $A$ is completely determined by its value on any basis of A.

Lemma 5.4. A weak fuzzy automaton homomorphism on $A$ is completely determined by its values on any basis of $A$.

Definition 5.5. Let $A_{i}=\left(Q_{i}, \Sigma, \mu_{i}\right) \ll A$ and let $f_{i}: A_{i} \rightarrow B$ , for each $i \in\{1,2, \ldots, n\}$. The extension of $f_{i}$ by $f_{j}$ is the function $f_{i} \vee f_{j}$ defined by :

If $f_{i}(r)=f_{j}(r), \forall r \in Q_{i} \bigcap Q_{j}$, then

$$
\left(f_{i} \vee f_{j}\right)(q)= \begin{cases}f_{i}(q) & \text { if } q \in Q_{i}, \\ f_{j}(q) & \text { if } q \in Q_{j} .\end{cases}
$$

Otherwise, $f_{i} \vee f_{j}$ is the empty function.

Note that $f_{i}$ is extendable to $A_{j}$ by $f_{j}$, if $f_{i} \vee f_{j}$ is a nonempty function. Further, $f_{i}$ is extendable to $A_{j}$, if there exists $g: A_{j} \rightarrow B$ such that $f_{i} \vee g$ is a nonempty function.

Theorem 5.6. If $f_{1}, f_{2} \in W E^{F}(A)$, then $f_{1} \vee f_{2} \in W E^{F}(A)$.

Lemma 5.7. The extension $\vee$, taken as a binary operation, is both commutative and associative; composition of extensions is the extension of respective compositions; the inverse of a fuzzy isomorphic extension is the extension of the respective inverses.

The following theorem decompose a weak fuzzy automaton homomorphism from $A$ to $B$ into weak fuzzy automaton homomorphism from primaries of $A$ to $B$. Hence we call it as a weak homomorphism decomposition theorem.

Theorem 5.8. Let $A$ and $B$ be fuzzy automata and let $f \in$ $W H^{F}(A \rightarrow B)$. If $\left\{A_{1}, A_{2}, \ldots A_{n}\right\}$ is a primary decomposition of $A$. Then $\mathrm{f}$ has a unique decomposition $f=\bigvee_{i=1}^{n} f_{i}$, where $f_{i} \in W H^{F}\left(A_{i} \rightarrow B\right)$.

Proof. Take $f_{i}=\left.f\right|_{A_{i}}, \forall i$. Then $f_{i} \in W H^{F}\left(A_{i} \rightarrow B\right), \forall i$. By Theorem 5.6, $f=\bigvee_{i=1}^{n} f_{i}$. Note that by Theorem 4.7, the decomposition of $f$ is unique (upto appearance).

Theorem 5.9. Let $\left\{A_{1}, A_{2}, \ldots, A_{n}\right\}$ and $\left\{B_{1}, B_{2}, \ldots, B_{m}\right\}$ be primary decompositions of $A$ and $B$ respectively. Let $\pi_{n}$ denotes the set of permutations of $\{1,2, \ldots, n\}$. Then 


$$
\begin{aligned}
1 W H^{F}(A \rightarrow B) & =\left\{\bigvee_{i=1}^{n} f_{i}: f_{i} \in W H^{F}\left(A_{i} \rightarrow B\right)\right\}^{+} \\
& =\left\{\bigvee_{i=1}^{n} f_{i}: f_{i} \in \bigcup_{j=1}^{m} W H^{F}\left(A_{i} \rightarrow B_{j}\right)\right\}^{+} \\
2 W E^{F}(A) & =\left\{\bigvee_{i=1}^{n} f_{i}: f_{i} \in W H^{F}\left(A_{i} \rightarrow A\right)\right\}^{+} \\
& =\left\{\bigvee_{i=1}^{n} f_{i}: f_{i} \in \bigcup_{j=1}^{n} W H^{F}\left(A_{i} \rightarrow A_{j}\right)\right\}^{+} \\
3 W I^{F}(A \rightarrow B) & =\bigcup_{\sigma \in \pi_{n}}\left\{\bigcup_{i=1}^{n} f_{i}: f_{i} \in W I^{F}\left(A_{i} \rightarrow B_{\sigma(i)}\right)\right\}^{+} \\
4 W G^{F}(A) & =\bigcup_{\sigma \in \pi_{n}}\left\{\bigcup_{i=1}^{n} f_{i}: f_{i} \in W I^{F}\left(A_{i} \rightarrow A_{\sigma(i)}\right)\right\}^{+}
\end{aligned}
$$

where $E^{+}$denotes the subset of all nonempty members of $E$, where $E$ is a set of extensions.

Proof. (1) By Theorem 5.8, any weak fuzzy automaton homomorphism on $A$ to $B$ is of the form $\bigvee_{i=1}^{n} f_{i}, f_{i} \in W H^{F}\left(A_{i} \rightarrow B\right)$. Conversely a nonempty extension $\bigvee_{i=1}^{n} f_{i}, f_{i} \in W H^{F}\left(A_{i} \rightarrow B\right)$, is a weak fuzzy automaton homomorphism from $A$ to $B$ as it preserves transitions on each primary and hence on the entire automaton.

By similar arguments we can prove other equalities.

We now return back, from deviation, to characterization of weak fuzzy automaton isomorphism.

Theorem 5.10. Let $f \in W E^{F}(A)$. Then $f \in W G^{F}(A)$ if and only if $O_{f}(p)=C_{f}(p)$, for each $\mathrm{p}$ in a generating set of $A$.

Proof. Suppose $f \in W G^{F}(A)$, then by Theorem 4.21, $O_{f}(p)=C_{f}(p), \forall p \in Q$ and hence for all $p$ in generating set of $A$. Conversely let $R$ be a generating set of $A$ and $O_{f}(p)=C_{f}(p), \forall p \in R$ where $f \in W E^{F}(A)$. Let $s, t \in Q$. Then there exist $p, q \in R$ and $x, y \in \Sigma^{*}$ such that $\mu(p, x, s)>$ 0 and $\mu(q, y, t)>0$. Let $\left|O_{f}(p)\right|=m$ and $\left|O_{f}(q)\right|=n$. Then $f(s)=f(t) \Rightarrow f^{m n}(s)=f^{m n}(t)=r$, say. Therefore $\mu\left(f^{m n}(p), x, r\right)>0$ and $\mu\left(f^{m n}(q), y, r\right)>0$. By circularity of orbits $\mu(p, x, r)>0$ and $\mu(q, y, r)>0$. Therefore $r=s=t$, as is $\mu$ a fuzzy function. Thus, $f$ is $1-1$. Since $Q$ is finite, $f$ is onto. Hence $f \in W G^{F}(A)$.

Theorem 5.11. Let $f \in W E^{F}(A)$. Then $f \in W G^{F}(A)$ if and only if $f$ maps basis of $A$ onto basis of $A$.

Proof. Suppose $f$ maps basis of $A$ onto basis of $A$. Let $R$ be a basis of $A$ and let $r \in Q$. Then by Theorem 4.7, $r \in Q_{S}$ for some primary $S$ of $A$. Let $q \in g e n S \cap f(R)$ and let $\mu(q, x, r)>0$. Then there exists $p \in R$ such that $f(p)=q$ and hence $f(s)=r$ where $\mu(p, x, s)>0$. Thus $f$ is onto. Since $Q$ is finite, $f$ is one one. Hence $f \in W G^{F}(A)$. Conversely suppose $f \in W G^{F}(A)$. Since any two bases of $A$ has same number of elements and $f \in W G^{F}(A), f$ maps basis onto basis of $A$.

Theorem 5.12. If there is a weak fuzzy automaton isomorphism $f$, between $A$ and $B$, then
(i) $W I^{F}(A \rightarrow B)=f\left(W G^{F}(A)\right)=\left(W G^{F}(B) f, \forall f \in\right.$ $W I^{F}[A \rightarrow B]$
(ii) $\left|\left(W G^{F}(A)\right)\right|=\left|W I^{F}(A \rightarrow B)\right|=\left|\left(W G^{F}(B)\right)\right|$.

Proof. By Theorem 4.16 and decomposition theorem 5.8, the proof follows.

Thus we have

$W G^{F}(A)=f^{-1}\left(W I^{F}(A \rightarrow B)\right)$ and
$W G^{F}(B)=\left(W I^{F}(A \rightarrow B)\right) f^{-1}, \forall f \in W I^{F}(A \rightarrow B)$

Theorem 5.13. Let $A=\left(Q_{A}, \Sigma, \mu\right)$ and $B=\left(Q_{B}, \Sigma, \gamma\right)$ be fuzzy automata. (i)There exists $f \in W H^{F}(A \rightarrow B)$ if and only if, for any ordered basis $R=\left(p_{1}, p_{2}, \ldots, p_{n}\right)$ of $A$, there exists an ordered $n$-tuple $T=\left(q_{1}, q_{2}, \ldots, q_{n}\right)$ of states of $B$ satisfying

$$
\begin{aligned}
& \forall p_{i}, p_{j} \in R, \forall y, z \in \Sigma^{*}, \mu\left(p_{i}, y, p\right)>0 \text { and } \mu\left(p_{j}, z, p\right)>0, \\
& \text { for some } p \in Q_{A} \Rightarrow \gamma\left(q_{i}, y, q\right)>0 \text { and } \gamma\left(q_{j}, z, q\right)>0, \\
& \text { for some } q \in Q_{B} \text {. }
\end{aligned}
$$

Moreover, $f\left(p_{i}\right)=q_{i}, \forall i \in\{1,2, \ldots, n\}$ defines $f$ on $Q_{A}$, where $f(s)=t$, if $\mu\left(p_{i}, w, s\right)>0$ and $\gamma\left(q_{i}, w, t\right)>0$, for some $p_{i} \in R$, and $w \in \Sigma^{*}$.

(ii) There exists $f \in W I^{F}(A \rightarrow B)$ if and only if , for any ordered basis $R=\left(p_{1}, p_{2}, \ldots, p_{n}\right)$ of $A$, there exists an ordered basis $T=$ $\left(q_{1}, q_{2}, \ldots, q_{n}\right)$ of $B$ satisfying

$\forall i, j \in\{1,2, \ldots, n\}, \forall y, z \in \Sigma^{*}, \mu\left(p_{i}, y, p\right)>0$ and $\mu\left(p_{j}, z, p\right)>0$, for some $p \in Q_{A} \Leftrightarrow \gamma\left(q_{i}, y, q\right)>0$ and $\gamma\left(q_{j}, z, q\right)>0$,

for some $q \in Q_{B}$.

$f\left(p_{i}\right)=q_{i}, \forall i \in\{1,2, \ldots, n\}$ defines $f$ on $Q_{A}$.

Proof. (i) Let $R$ and $T$ exists and satisfy $(1$. Define $f: A \rightarrow$ $B$ by $f\left(s_{i}\right)=t_{i}$, if $\mu\left(p_{i}, w, s_{i}\right)>0$ and $\gamma\left(q_{i}, w, t_{i}\right)>0$, for some $w \in \Sigma^{*}$. Since (1) is satisfied, $\mu\left(p_{i}, y, p\right)>0$ and $\mu\left(p_{j}, z, p\right)>0$, for some $p \in Q_{A}$, we have $\gamma\left(q_{i}, y, q\right)>0$ and $\gamma\left(q_{j}, z, q\right)>0$,for some $q \in Q_{B}$. Thus $f(p)=q$. i.e. $f$ is well defined. Let $\mu\left(p_{i}, u, r\right)>0$ for some $p_{i} \in R$ and $u \in \Sigma^{*}$. Let $r_{1} \in T$ and $x \in \Sigma^{*}$ with $\mu\left(r, x, r_{1}\right)>0$. Then $\mu\left(p_{i}, u x, r_{1}\right)>0$ implies that $\gamma\left(q_{i}, x, t_{1}\right)>0$ for some $t_{1} \in Q_{B}$. Hence $f$ is weak fuzzy automaton homomorphism. By Lemma 5.4,it is immediate that $f\left(p_{i}\right)=q_{i}, \forall i \in\{1,2, \ldots, n\}$ defines $f$ on $Q_{A}$. Converse can be proved by using Theorem 5.8. Similarly (ii) can be proved.

\section{Conclusion}

Apart from introduction of various concepts such as perfect fuzzy automaton, singly generated fuzzy automaton, primaries, state fuzzy equivalent relation, state fuzzy endomorphic (automorphic) set and basis of a fuzzy automaton, precisely following conclusions are drawn.

(1) The set $W G^{F}(A)$, of all fuzzy automaton isomorphisms of a fuzzy automaton $A$, forms a group.

(2) In the case of strongly connected fuzzy automaton, if two fuzzy automaton homomorphisms coincides at a state, then they are identical.Further, $\left|W G^{F}(A)\right| \leq|Q|$.

(3) We have characterized weak fuzzy automaton homomorphisms (and isomorphisms) on perfect fuzzy automata and obtained the equality between $\left|W G^{F}(A)\right|$ and $|Q|$. In fact, $W G^{F}(A)$ is homomorphic image of a monoid $\Sigma^{*}$ over any perfect fuzzy automaton $A=(Q, \Sigma, \mu)$.

(4) Arbitrary fuzzy automaton is decomposed into its distinct primaries, that are maximal singly generated fuzzy automaton.

(5) Therefore, we have characterized the weak fuzzy automaton homomorphism (and isomorphism) in terms of the state-fuzzy equivalence relation over singly generated fuzzy automata.

(6) Every weak fuzzy automaton homomorphism on an arbitrary fuzzy automaton is determined by its values on any of its basis.

(7) A weak fuzzy automaton homomorphism is an isomorphism if and only if it maps basis onto basis. 
(8) Every weak fuzzy automaton homomorphism on an arbitrary fuzzy automaton is obtained by join $(\vee)$ of weak fuzzy automaton homomorphisms on its all primaries.

\section{Acknowledgment}

The first author would like to thank University Grant Commission, New Delhi, India for the financial support under the scheme FIP.

\section{REFERENCES}

[1] Bavel Z., Structure and transition-preserving functions of $f$ nite automata, J. ACM, 15(1), pp 135-158,(Jan.1968).

[2] Cho S.J., Kim J.G.,Tae Kim S., T-fuzzy semiautomata over finite group, Fuzzy Sets and Systems, 108, pp 341-351,(1999).

[3] Choubey A., Ravi K.M., Minimization of deterministic finite automata with vague (final) states and intutionistic fuzzy ( $f$ nal) states, Iranian Journal of Fuzzy Systems, 10(1), pp 7588,(2013).

[4] Ciric Miroslav, Aleksandar Stamenkovic, Jelena Ignjatovic, Tatjana Petkovic, Fuzzy relation equations and reduction of fuzzy automata, J. Comput. Syst. Sci., 76(7), pp 609633,(2010).

[5] Das P.,On some properties fuzzy semiautomaton over a finite group, Information Sciences, 101, pp 71-84,(1997).

[6] Fleck A.C., Isomorphism groups of automata, J. ACM, 9(4), pp 469-476,(Oct.1962).

[7] Fleck A.C., On the automorphism group of an automaton, J. ACM, 12(4), pp 566-569,(Oct.1965).

[8] Jianhua Jin, Qingguo Li, Yongming Li, Algebraic properties of L-fuzzy finite automata, Information Sciences, 234, pp 182202, (2013).

[9] Kim Y. H., Kim J. G. and Cho S. J. Product of T-generalized state machines and T-generalized transformation semigroups, Int. Journal of Fuzzy Sets and Systems 93, pp 87-97,(1998).

[10] Kumbhojkar H. V. and Chaudhari S. R. , On proper fuzzificztion of finite state machine, Int. Journal of Fuzzy Mathematics, 8(4), pp 1019-1027,(December 2000).

[11] Kumbhojkar H. V. and Chaudhari S. R., On covering of products of fuzzy finite state machines, Fuzzy Sets and Systems, 125, pp 215-222,(2002)

[12] Malik D. S., Mordeson J. N. and Sen M. K., Product of fuzzy finite state machines, Int. Journal of Fuzzy Sets and Systems 92, pp 95-102,(1997).

[13] Malik D.S., Mordeson J.N.,Sen M.K.,On subsystems of fuzzy finite state machines, Fuzzy Sets and Systems, 68, pp 8392,(1994).

[14] Mo Zhi-wen, HU Hong-li, Minimization of fuzzy finite generalized automata, J. of Electronic Science and Technology of China, 4(1), pp 86-88,(2006).

[15] J.Mockor, Semigroup homomorphisms and fuzzy automata, Soft Computing, 6 (6), pp 422-427,(2002).

[16] Mordeson J. N. and Malik D. S.(2002), Fuzzy automata and languages: theory and applications, Chapman and Hall / CRC, Boca Raton, London.

[17] Tatjana Petkovic, Congruences and homomorphisms of fuzzy automata, Fuzzy Sets and Systems, 157, pp 444-458,(2006).

[18] Tiwari S. P. and Srivastava A. K.,On a decomposition of fuzzy automata, Fuzzy Sets and Systems, 151(3), pp 503$511,(2005)$.
[19] Wegg G.P., The structure of an automaton and its operation preserving transformation group, J. ACM, 9(3), pp 345349,(July.1962).

[20] Young Bae Jun, Intutionistic fuzzy finite state machines, J. Appl. Math. and Computing, 17, pp 109-120,(2005). 